\title{
Una Aproximación Conceptual a la Educación para la Justicia Social y Ambiental
}

\section{A Conceptual Approach to Education for Social and Environmental Justice}

\author{
Sergio Carneros * \\ F. Javier Murillo \\ Irene Moreno-Medina \\ Universidad Autónoma de Madrid, España
}

\begin{abstract}
Justicia Social y Justicia Ambiental son dos enfoques fuertemente entrelazados que pueden considerarse solo uno. En este artículo abordamos la conceptualización del termino Justicia Social y Ambiental, como un todo, a partir de la revisión de las cuatro grandes concepciones que sobre el mismo existen y que conviven en la actualidad: Justicia Social y Ambiental como Distribución, desde la Teoría de las Capacidades, como Reconocimiento y Participación. A partir de esas ideas se llega a la formulación de Justicia Social y Ambiental como la consecución del reconocimiento, la participación y la distribución de recursos materiales, culturales y ambientales (tanto perjudiciales como beneficiosos) de forma plena y equitativa, todo ello bajo un criterio intrageneracional, intergeneracional, interespecies e internacional que permita el desarrollo de una vida digna. De este concepto se desarrolla una idea de Educación para la Justicia Social y Ambiental multidimensional conformada por cuatro elementos: Educación equitativa, educación, crítica, educación democrática y educación para el desarrollo sostenible (o ambiental).
\end{abstract}

Descriptores: Justicia social; Educación para el desarrollo sostenible; Educación ambiental; Democracia; Ética ambiental.

Social Justice and Environmental Justice are two strongly intertwined approaches that can be considered only one. In this paper, we address the conceptualization of the term of Social and Environmental Justice, as a whole, based on the review of four main concepts about it and that coexist today: Social and Environmental Justice as Distribution, from the Theory of Capacities, such as Recognition and Participation. Based on these ideas, Social and Environmental Justice is considered as the achievement of the recognition, participation and distribution of material, cultural and environmental resources (both harmful and beneficial) in a full and equitable manner, all under a criterion intragenerational, intergenerational, interspecies and international that allows the development of a dignified life. This concept develops an idea of Education for Social and Environmental Justice which is multidimensional and it's made from four elements: Equitable education, critic education, democratic education and education for sustainable (or environmental) development.

Keywords: Social justice; Education for sustainable development; Environmental education; Democracy; Environmental ethics.

*Contacto: sergio.carneros@hotmail.com

ISSN: 2254-3139

www.rinace.net/riejs/

revistas.uam.es/riejs
Recibido: 9 de noviembre 2017

$1^{\text {a }}$ Evaluación: 21 de diciembre 2017

Aceptado: $\quad 10$ de marzo 2018 


\section{Introducción}

La Justicia Social y la Justicia Social son dos enfoques tan directamente entrelazados e interdependientes entre sí que quizá deba ser solo uno (Grass, 1995; Haughton, 1999). Así, podríamos hablar de "Justicia Ecosocial”, o bien usar "Justicia Ambiental” o "Justicia Social”, absorbiendo el término elegido los aspectos que el otro aborda. Sin embargo, creemos que hablar de Justicia Social y Ambiental es los más adecuado dado que se visibiliza cada uno de los elementos, respetando su origen diferenciado.

Pero no es posible referirse a la Justicia Social y Ambiental sin relacionarlo con el término "sostenibilidad", palabra asociada comúnmente con los movimientos ambientales. No solo se trata de atender al presente, sino también de mirar a futuras generaciones y a otras especies. Como señala Agyeman (2005), es necesario que la Justicia se desplace al centro del escenario del discurso de la sostenibilidad, para que haya una oportunidad real de futuro.

En este artículo comenzaremos describiendo las grandes concepciones de Justicia Social y Ambiental que existen y que conviven en la actualidad:

- Justicia Social y Ambiental como Distribución (Angyeman, 2007; Rawls, 1979, 2002).

- Justicia Social y Ambiental desde la Teoría de las Capacidades (Nussbaum, 2007; Sen, 2010).

- Justicia Social y Ambiental como Reconocimiento (Collins, 1991; Fraser, 2008; Fraser y Honneth, 2006; Honneth, 2007).

- Justicia Social y Ambiental como Participación (Fraser, 2008; Young, 2000a, 2011).

A partir de los cuales nos atreveremos a proponer una definición de Justicia Social y Ambiental y, con ello, abordaremos la conceptualización de la Educación para la Justicia Social y Ambiental.

\section{Justicia social y ambiental como distribución}

La Justicia distributiva se basa, inicialmente, en el modo en que los bienes primarios y los costes y beneficios ambientales se encuentran distribuidos en la sociedad (Agyeman, 2007; Beauchamp, 2001; Rawls, 1979). Jost y Kay (2010) y North (2006) insisten en que el punto de interés está en desarrollar principios de Justicia que permitan corregir la privación de recursos y que estos sean mejor distribuidos en función de los distintos intereses.

Utilizamos la clasificación de Justicia Ecológica de Bosselmann (2006) para abordar la Justicia Social y Ambiental como distribución. Esta hace referencia a tres tipos de Justicia: Justicia Intrageneracional (dimensión entre una misma generación), Justicia Intergeneracional (perspectiva de futuras generaciones) y Justicia Interespecies (preocupación por el mundo natural no humano).

\subsection{Justicia intrageneracional}

Aborda la dimensión social y ambiental dentro de cada generación para alcanzar la Justicia. A partir de Murillo y Hernández-Castilla (2011), señalamos los principios que delimitan la distribución de los beneficios y los lastres en la sociedad: 
- Justicia igualitaria: a cada persona una parte igual. En cuestiones de ambiente significaría "iguales porciones de espacio ambiental para todos y cada uno de los seres humanos" (Riechmann, 2003, p. 8). Aunque esta idea puede resultar inicialmente sencilla, la principal dificultad que entraña es que las personas comienzan con diferentes beneficios y desventajas. Además, no son iguales en todos los aspectos, de modo que la igualdad en la distribución conlleva desigualdades inmerecidas.

- Justicia según la necesidad: a cada persona de acuerdo con sus necesidades individuales. De tal forma que los que tienen más necesidades de un bien deben poseer asignaciones mayores. Este principio demanda una redistribución de los bienes en función de la necesidad para satisfacer las necesidades humanas básicas e impedir que las personas vivan en condiciones de desventaja social y ambiental significativas por causas ajenas a su voluntad. Aquí introduciremos el Principio de Diferencia, según el cual las desigualdades solo se pueden justificar si benefician a los más desaventajados, ya que de lo contrario no son lícitas (Rodríguez Zepeda, 2009). Rawls (2002) entiende que éste es un principio de compensación y de políticas de discriminación positiva necesario para que las desigualdades naturales o de nacimiento sean compensadas.

- Justicia según el mérito: a cada persona según sus méritos. Según este planteamiento los que más contribuyen a la generación de beneficios sociales y de riqueza deben tener también una mayor proporción de los mismos (Beauchamp, 2001).

\subsection{Justicia intergeneracional}

Tanto Bosselmann (2006) como Rawls (1979) dan importancia a la Justicia intergeneracional, pues sin su existencia no podría haber Justicia en la sociedad ya que habría privilegios para las generaciones actuales en detrimento de las generaciones futuras (Wissenburg, 1999). Este principio exige que los derechos deben ser distribuidos de tal manera que se mantengan disponibles para una futura redistribución. En otros términos, consiste en la obligación de no destruir bienes en aquellos casos en que sean irreemplazables (Hervé, 2010; Wissenburg, 1999). Por tanto, puesto que no sabemos qué necesitarán las futuras generaciones se trata de mantener la integridad ambiental (Bosselmann, 2006). A su vez, es importante señalar cómo la estructura cultural heredada debe ser transmitida al menos en un estado igualmente rico que aquel en el que la hemos encontrado (Dworkin, 1985; Gosseries, 2012).

Weiss (1999) señala la existencia de tres principios de equidad intergeneracional:

- Conservación de las opciones: Una generación debe tener, al menos, el mismo número de opciones que la precedente.

- Conservación de la calidad: La calidad del medio debe tener las mismas condiciones o mejores para las siguientes generaciones.

- Conservación del acceso: Los miembros de la actual generación deben poder acceder sin discriminaciones a los recursos naturales y culturales del planeta, manteniendo el respeto hacia las futuras generaciones.

Entonces, ¿quién debe hacer este esfuerzo para hacer un mundo digno para las futuras generaciones? La Justicia Intergeneracional también muestra preocupación por el sacrificio de los menos favorecidos de la generación presente con el fin de prevenir 
mayores injusticias futuras o, simplemente, de hacer posible un futuro mejor (Dworkin, $1985)$.

\subsection{Justicia interespecies}

Esta idea está relacionada con la preocupación por el mundo natural no humano. La Justicia no tiene que ver tanto con la distribución justa entre la población humana como entre ésta y el resto de los seres vivos con los que compartimos la biosfera (Mosterín y Riechmann, 1995; Riechman, 2000, 2003). Esto mismo es reconocido por el Movimiento de Justicia Ambiental estadounidense en los años noventa, quedando plasmado en el primero y tercero de los diecisiete "Principios de Justicia Ambiental" que se aprobaron en el First National People of Color Environmental Leadership Summit:

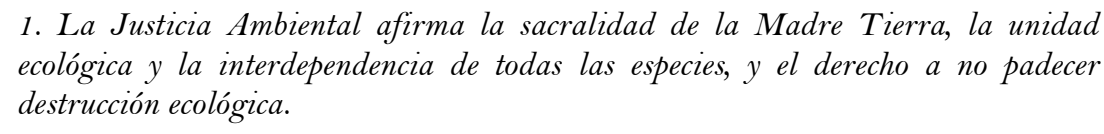

1. La Justicia Ambiental afirma la sacralidad de la Madre Tierra, la unidad ecológica y la interdependencia de todas las especies, y el derecho a no padecer destrucción ecológica.

3. La Justicia Ambiental fundamenta el derecho a usos éticos, equilibrados y responsables de la tierra y los recursos renovables, en pro de un planeta sostenible para los seres humanos y las demás criaturas vivas. (Hofrichter, 1994, p. 237)

Por tanto, la distribución debe ser entendida no entre humanos sino entre seres vivos (Riechmann, 2003; Riechmann et al., 1995). Como señala el poeta estadounidense Gary Snyder (1995, p. 60), "incluso si se lograse la Justicia social y económica para toda la gente, seguiría existiendo una necesidad drástica de Justicia ecológica, lo que significa dejar mucha tierra y agua para que los seres no humanos puedan vivir su vida".

Una vez descritos los niveles de Justicia que tenemos en cuenta en la distribución (intergeneracional, intrageneracional y interespecies), nos debemos preguntar qué distribuir. Para Rawls (2002), lo que debe estar distribuido son los bienes primarios, es decir lo que necesita la ciudadanía como personas libres e iguales, y propone una lista de esos bienes primarios:

- Derechos y libertades básicas.

- Libertad de desplazamiento y de elección de ocupación.

- Poderes y prerrogativas de los puestos y cargos de responsabilidad en las Instituciones políticas y económicas.

- Ingreso y riqueza.

- Bases sociales de respeto a sí mismo.

Según Rawls (1993), esta lista puede ampliarse si respeta el límite de la Justicia como imparcialidad y el límite de la simplicidad y disponibilidad de información. Sin embargo, la ampliación de la lista también debe respetar el espíritu de los cinco bienes enumerados, y que no son fines, sino medios (Murillo y Hernández-Castilla, 2011).

Por tanto, Rawls deja la puerta abierta a que entren desde la Justicia Ambiental otros aspectos que deben distribuirse. Algunos ejemplos son (Angyeman, 2007; Hofrichter, 1994; Mosterín y Riechmann, 1995; Riechmann, 2003):

- Degradación y coste ambiental (residuos tóxicos, contaminación...).

- Espacio.

- Energía. 
- Beneficios ambientales.

Rawls (1979), define los fundamentos de un nuevo liberalismo y desencadena la polémica que todavía hoy continúa entre liberales de distinto signo y comunitarios de diverso tipo (Cortina, 1993). Benedicto (2010) señala que dentro de la tradición liberal podemos encontrar autores/as que se sitúan en una línea de liberalismo igualitario o social como Rawls (1993) o Dworkin (1993) y también otros que defienden la radical independencia del individuo proponiendo un liberalismo libertario o individual como Nozick (1988) o Hayek (1948).

Las críticas presentadas por el pensamiento comunitarista, representado por Macintyre (1984), Sandel (1982), Taylor (2003) o Walzer (2001), han contribuido a la redefinición hecha por Rawls de la relación entre la persona moral, la ciudadanía y la comunidad política, y también a las posteriores modificaciones que otros/as autores/as como Dworkin (1993), Nagel (1991) o Raz (1986) han incorporado a la tradición liberal.

Para definir la corriente comunitarista, Valcárcel (2002, p. 116) señala que los "comunitaristas son aquellos autores que, fundamentalmente, sostienen que los derechos individuales han de ceder, en ciertos casos, ante los derechos de la comunidad; y que con ello la moralidad del conjunto -incluida una práctica mejor de la individualidadaumenta”. Aunque es una definición algo genérica, los comunitaristas como Macintyre (1984), observan que las sociedades en las que los derechos individuales parecen estar asegurados, no son capaces de evitar la insolidaridad y el debilitamiento de los lazos comunitarios (Benedicto, 2010).

Otra crítica hacia Rawls (1979) la realizan desde la Teoría de las Capacidades de Sen (1995, 2010) y Nussbaum (2002, 2007). Plantean que es necesario centrarse, no en la riqueza ni en los bienes, sino en las capacidades de las personas. Debido a su gran popularidad lo expondremos a continuación de manera más detenida.

\section{Justicia social y ambiental desde la teoría de las capacidades}

El economista indio Amartya Sen critica la teoría de la Justicia distributiva antes descrita, por ser demasiado instrumental. En su texto Inequality reexamined (Sen, 1992), plantea que la idea de la Justicia se encuentra en las libertades reales que gozan los individuos. Esta es la diferencia entre Sen y Rawls. Mientras este último se centra en los medios para lograr los fines deseados por los individuos, Sen fija su atención en la libertad real de las personas, concebida como la capacidad de lograr realizaciones, lo que constituye un fin para cada persona (Murillo y Hernández-Castilla, 2011).

Sen (1992) sostiene que la atención en las posesiones de medios para la libertad (como, por ejemplo, los bienes primarios) no pueden proporcionar las comparaciones interpersonales que constituyan una base informacional de la Justicia. Por tanto, las exigencias individuales han de ser consideradas no por los medios, sino por las libertades que gozan realmente para elegir entre alternativos modos de vida (Sen, 1992, 2010).

Sen (1992) considera que el enfoque de Rawls es insuficientemente igualitario. Los bienes primarios y los recursos son importantes como medios para obtener funcionamientos importantes, pero el problema de concentrarnos en estos instrumentos es el "olvido" que 
se realiza, al no tener en cuenta las distintas capacidades de los individuos para transformarlos en funcionamientos (Murillo y Hernández-Castilla, 2011). Sen señala de forma explícita:

Los recursos de que dispone una persona o los bienes que alguien tiene pueden ser indicadores muy imperfectos de la libertad de que realmente disfruta la persona para hacer esto o ser aquello (...). El cambio de atención en la filosofía politica contemporánea, tal como ocurre en las teorías de Rawls, hacia comparaciones interpersonales basadas en los recursos puede verse claramente como un paso hacia una mayor atención a la libertad. Pero estos cambios son esencialmente insuficientes. (Sen, 1995, p. 51)

Sen $(1992,2010)$ exige que examinemos el valor de los procedimientos y las capacidades, en vez de atender solo a los medios necesarios para tales realizaciones y libertades.

Una visión complementaria es aportada por la profesora estadounidense Martha Nussbaum $(2002,2007)$ desde la filosofía política. Esta autora parte, como Sen, de las ideas de Rawls, cuyos planteamientos se consideran como la teoría política más sólida y útil para abordar los problemas contemporáneos. Sin embargo, sus aportaciones nacen como crítica al contractualismo, situado en la base de los planteamientos de rawlianos (Murillo y Hernández-Castilla, 2011). Nussbaum (2007) aborda esa crítica a partir de tres elementos: la Justicia hacia las personas con discapacidad, al tratamiento de los animales no humanos (que nosotros hemos incluido anteriormente a través de la Justicia interespecies) y a las relaciones internacionales. Respecto a este último punto, Nussbaum (2007) señala:

\footnotetext{
Cualquier teoría de Justicia que pretenda ofrecer una base para que todos los seres humanos tengan unas oportunidades de vida decentes debe tener en cuenta tanto las desigualdades internas de cada país como las desigualdades entre países, y debe estar preparada para abordar las complejas intersecciones de estas desigualdades en un mundo cada vez más interconectado. (p. 301)
}

Respecto a la Justicia hacia las personas con discapacidad, Nussbaum crítica los planteamientos de Rawls (1979), según los cuales los individuos que participan en la elección de los principios de Justicia en la posición original han ser independientes y tener cierta igualdad de facultades. Nussbaum (2007) defiende que este procedimiento es injusto hacia las personas con discapacidad. Alega que las personas en situación de discapacidad mental no podrían formar parte de la deliberación original, quedando como ciudadanía de segunda. Para que las necesidades de las personas con discapacidad estén presentes desde el principio, así como la simpatía, la sociabilidad y la preocupación por los otros, es necesario no concebir la Justicia como el resultado de un pacto, sino a partir del bienestar de los individuos y la atención a sus necesidades y capacidades más básicas (Murillo y Hernández-Castilla, 2011; Nussbaum, 2004). De ahí que la esencia del enfoque de las capacidades considere las dimensiones fundamentales de la vida de las personas como el criterio fundamental de la Justicia. A partir de este planteamiento, Nussbaum (2002) presenta una lista de "diez capacidades funcionales humanas centrales":

1. Vida. Ser capaces de vivir una vida humana de duración normal, sin morir prematuramente o antes de que la vida se reduzca a algo que no merezca la pena vivir.

2. Salud corporal. Ser capaces de gozar de buena salud, estar adecuadamente alimentado y tener una vivienda digna. 
3. Integridad corporal. Ser capaces de moverse libremente y de forma segura de un lugar a otro; tener oportunidades para disfrutar de la satisfacción sexual y de la capacidad de elección en materia de reproducción.

4. Sentidos, imaginación y pensamiento. Ser capaces de utilizar los sentidos, de imaginar, pensar y razonar.

5. Emociones. Ser capaces de tener vínculos afectivos con cosas y personas ajenas. Poder desarrollarse emocionalmente sin las trabas de los miedos y ansiedades abrumadoras, ni por casos traumáticos de abusos o negligencias.

6. Razón práctica. Ser capaces de formar un concepto del bien e iniciar una reflexión crítica respecto de la planificación de la vida. Esto supone la protección de la libertad de conciencia.

7. Afiliación. Ser capaces de vivir con otros individuos y de volcarse hacia ellos. Recibir un trato como seres dignos cuyo valor es idéntico al de los demás. Esto implica, como mínimo, la protección contra la discriminación por motivo de raza, sexo, orientación sexual, religión, casta, etnia u origen nacional.

8. Otras especies. Ser capaces de apreciar y vivir en relación con los animales, las plantas y el mundo de la naturaleza.

9. Capacidad para jugar. Ser capaces de reír, jugar y disfrutar de actividades de ocio.

10. Control sobre el entorno de cada uno. Tanto político como material.

Nussbaum (2002) señala que la lista sigue estando abierta. Al respecto Hervé (2010) indica que es necesario recurrir e incluir elementos propios de Justicia Ambiental. Sin embargo, vemos que este enfoque de capacidades incluye aspectos relacionados con todos los ámbitos del ser humano y también con aspectos sociales y ambientales.

\section{Justicia social y ambiental como reconocimiento}

Otro gran planteamiento es lo que se ha llamado la Justicia Relacional (o cultural), definido como ausencia de dominación cultural, no reconocimiento e irrespeto (Fraser, 1997).

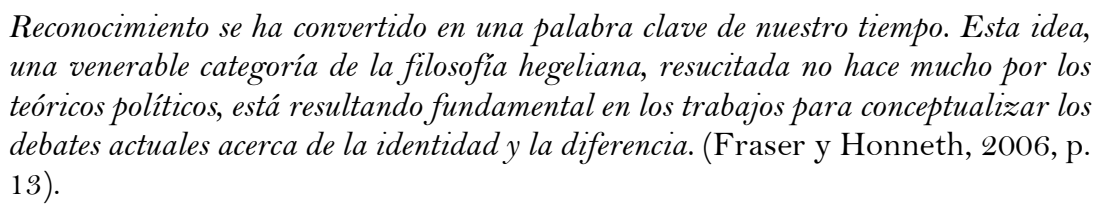
una venerable categoría de la filosofía hegeliana, resucitada no hace mucho por los teóricos políticos, está resultando fundamental en los trabajos para conceptualizar los debates actuales acerca de la identidad y la diferencia. (Fraser y Honneth, 2006, p. 13).

Fraser (1997) señala que las reivindicaciones de Justicia en el mundo actual parecen dividirse en dos tipos cada vez más claros. El primero y que ya hemos comentado, hace referencia a las de distribución, reivindicando una redistribución más justa de bienes y recursos. El segundo son las políticas de reconocimiento, donde el objetivo es un mundo que acepte las diferencias, en donde no haya que asimilar las normas culturales dominantes o de la mayoría.

Hervé (2010) indica que las situaciones de injusticia ambiental se producen respecto de grupos y comunidades antes que individuos. Y señala que "el reconocimiento apunta a la valorización de ciertas comunidades o grupos vulnerables de la sociedad y, a su vez, a la valorización de la naturaleza y de los ecosistemas en sí mismos” (Hervé, 2010, p. 21). 
La valoración de las minorías étnicas, raciales y sexuales, así como de la naturaleza y de los ecosistemas, intenta desarrollar un nuevo paradigma de la Justicia que sitúa al reconocimiento en su centro. El ascenso de la política de identidad ha cambiado el foco de las reivindicaciones de la redistribución. Consiste, por tanto, en idear una orientación que integre lo mejor de la política de la redistribución y lo mejor de la política del reconocimiento (Murillo y Hernández-Castilla, 2011).

Murillo y Hernández-Castilla (2011) señalan que las diferencias entre esta concepción de Justicia como reconocimiento y de la Justicia como distribución son resumidas por la propia Fraser (1997) en cuatro elementos:

1. Los dos enfoques asumen concepciones diferentes de injusticia. La estructura económica de la sociedad (marginación, explotación y privación); o injusticias culturales vinculadas a procesos de representación, interpretación y comunicación.

2. Proponen diferentes tipos de soluciones. En el enfoque de la distribución el remedio de la injusticia es algún tipo de reestructuración económica. Mientras que en el enfoque del reconocimiento la solución es el cambio cultural o simbólico o la reevaluación ascendente de las identidades no respetadas o sus productos culturales.

3. Asumen concepciones diferentes de las colectividades que sufren la injusticia. En el enfoque de la redistribución, las injusticias se producen sobre clases o colectividades definidas por el mercado o los medios de distribución. Mientras que desde el enfoque del reconocimiento están relacionadas con el género, la sexualidad, la cultura, la religión, etcétera.

4. Las diferencias de grupo se ven desde el enfoque de la distribución como diferenciales de injusticia, ligadas a estructuras socialmente injustas y por tanto se deben abolir. Frente al enfoque interpretativo, que está vinculado a una jerarquía de valores y por tanto requieren reevaluar los rasgos devaluados.

Fraser (1997) señala que la concepción del reconocimiento trae consigo problemas como la reificación de las identidades de los grupos, al simplificar o hacer desaparecer las diferencias internas, o el desplazamiento de los problemas relativos a la redistribución de bienes y riqueza que quedan relegados a un segundo plano. Por esto último, es importante tener en cuenta que las injusticias pueden ser claramente bidimensionales como la raza o la clase social. En este sentido, se reflexiona sobre una "tercera vía", donde la redistribución y el reconocimiento convergen a causa de la aceleración de la globalización económica y del descentramiento del marco nacional como referencia (Fraser y Honneth, 2006).

Fraser (Fraser y Honneth, 2006) defiende la búsqueda de un enfoque integrado que pueda reparar a la vez el reconocimiento erróneo y la mala distribución. Esta autora propone dos enfoques: reparación transversal y conciencia de los límites. La reparación transversal consistiría en:

(...) utilizar medidas asociadas con una dimensión de la Justicia para remediar desigualdades asociadas con la otra, es decir, utilizar medidas distributivas para reparar el reconocimiento erróneo y medidas de reconocimiento para reparar la mala distribución. La reparación transversal explota la imbricación de estatus y clase social con el fin de mitigar ambas formas de subordinación al mismo tiempo. (Fraser y Honneth, 2006, p. 80) 
Iglesias (2012) indica que el problema que plantea esta forma de reparación es que no podría usarse de manera sistemática ni generalizada. Por otro lado, esta autora señala que la conciencia de los límites no será otra cosa que "la conciencia del impacto de diversas reformas sobre los límites del grupo" (Fraser y Honneth, 2006, p. 82). Queda pendiente encontrar un esquema exitoso, pero de una manera o de otra, tal y como señala Fraser, "solo si buscamos enfoques integradores que unan redistribución y reconocimiento podremos satisfacer los requisitos de una justicia para todos" (Fraser y Honneth, 2006, p. 88).

\title{
4. Justicia social y ambiental como participación
}

La Justicia como Participación o Representación (en este trabajo utilizamos en mayor medida el término "participación") implica la promoción del acceso y la equidad para asegurar la plena participación en la vida social, especialmente para aquellos grupos que han sido sistemáticamente excluidos sobre la base de su etnia, edad, género, habilidad física o mental, educación, orientación sexual, situación socioeconómica u otras características (Bell, 2007; Harnett, 2001).

En este sentido, esta idea de Justicia se fundamenta en que no es suficiente con el mero reparto de bienes materiales, sino que también resulta imperativo difundir otros "bienes" asociados como la igualdad de oportunidades, el acceso al poder, la posibilidad de participar en diferentes espacios públicos o el acceso al conocimiento. Para conseguirlo es necesario entender la Justicia como un procedimiento o proceso en la medida que se convierte en una herramienta para lograr una justicia distributiva y un reconocimiento político (Murillo y Hernández-Castilla, 2011).

\begin{abstract}
La representación está ya siempre inherentemente presente en cualquier reivindicación de redistribución o de reconocimiento. La dimensión política está implícita en, y en realidad requerida por, la gramática del concepto de Justicia. De manera que no hay redistribución ni reconocimiento sin representación. (Fraser, 2008, p. 49)
\end{abstract}

Para Axel Honneth existe un vínculo claro entre la ausencia de respeto y reconocimiento y la falta de participación en la comunidad y sus instituciones (Fraser y Honneth, 2006). De modo que la ciudadanía sin participación se encuentra directamente y estructuralmente excluida de determinados derechos otorgados por la sociedad. El hecho de experimentar la negación de estos derechos conlleva la falta de consideración propia y de respeto hacia uno mismo (Murillo y Hernández-Castilla, 2011).

Honneth (2001) propone que la democracia sea entendida como una forma reflexiva de cooperación comunitaria que articule deliberación racional y comunidad democrática. Para este autor, la cooperación es el fundamento de todo tipo de sociabilidad, siendo el eslabón que articula autonomía personal y gobierno político.

Young (2000a, 2000b) defiende un procedimiento democrático como condición básica de la Justicia y considera que se necesita profundizar sobre la eliminación de la opresión y dominación institucional.

Para que una norma sea justa, todo el mundo que la aplica debe tener la oportunidad de ser considerado con una voz eficaz y debe tener la posibilidad de estar de acuerdo con ella sin coacción. Para que una condición social sea justa, debe permitir a todos satisfacer sus necesidades y ejercer su libertad; asi la Justicia requiere que todos puedan expresar sus necesidades. (Young, 2000b, p. 125) 
Por tanto, cualquier enfoque sobre Justicia debe estar centrado en los procesos políticos, pues estos conllevan gran variedad de injusticias, tanto en la distribución de bienes como en la distribución del reconocimiento. Insiste en situar a la Justicia en las normas y los procedimientos de acuerdo con el lugar donde se toman las decisiones (Murillo y Hernández-Castilla, 2011; Trejo Pérez, 2016).

Iris MarianYoung (2000a, 2000b) aboga por un modelo de democracia comunicativa donde la participación necesita ejercerse en diferentes instituciones tanto sociales como culturales, en contextos políticos y de gobierno. Y Fraser (1997), con un objetivo similar, defiende una democracia radical entendida como el sistema político que garantice los derechos sociales y la igualdad de oportunidades para participar en la esfera pública (Avedaño, 2010).

La exigencia de mayor voz y poder para los individuos y las comunidades ha sido siempre parte esencial del movimiento ambiental. La original definición del racismo ambiental en Estados Unidos planteada por Chavis (1993) incluía, como parte de su justificación, las prácticas excluyentes y restrictivas que limitan la participación de la gente de color que se daban en las juntas donde se toman decisiones, en las comisiones y en los entes reguladores (Bullard, 1993a, 1993b).

Como ejemplo, en los 10 Principles o Just Climate change Policies del Environmental Justice and Climate Change Initiative (EJCC) se señala que "A todos los niveles y en todos los ámbitos, las personas deben tener voz y voto en las decisiones que afecten a sus vidas. Quienes toman las decisiones deben incluir a las comunidades en el proceso político" (Hoerner y Robinson, 2008, p. 56).

El derecho a la participación y el acceso a la información es quizá uno de los aspectos más universalmente aceptados. La participación es el mecanismo o procedimiento necesario para lograr una mejor distribución y un mayor reconocimiento. Por tanto, una teoría de la Justicia debe focalizarse en el proceso democrático, que permite establecer estructuras para hacer frente tanto a los problemas de distribución como de reconocimiento (Hervé, 2010; Schlosberg, 2007).

\section{Hacia una definición de justicia social y ambiental}

En la revisión que llevamos realizada hasta ahora sobre el concepto de Justicia Social y Ambiental, podemos ver su complejidad, su evolución en el tiempo, su carácter multidimensional y multidisciplinar y su fuerte componente ideológico. Como hemos visto, el término Justicia Social y Ambiental tiene un gran carácter distributivo ya que su argumento básico se refiere a la preocupación por cómo los bienes primarios y los impactos ambientales, se distribuyen entre los distintos miembros de la sociedad.

La obra "Teoría de la Justicia" (Rawls, 1979) marca un antes y un después en el devenir de ese concepto. La mayoría de especialistas están de acuerdo en esta distribución, pero qué distribuir es uno de los debates abiertos en estos años: de bienes primarios como decía Rawls, de recursos (Dworkin, 1981), de los impactos medioambientales, tanto positivos como negativos (Agyeman y Evans, 2004; Heiman, 1996), o priorizando en un enfoque desde las capacidades (Nussbaum, 2002; Sen, 2010). Y a su vez cómo realizarlo, si teniendo en cuenta al individuo (Dworkin, 1981; Nozick, 1988), a la comunicad (Macintyre, 1984; Sandel, 1982; Taylor, 2003; Walzer, 2001) o a todas las especies (Mosterín y Riechmann, 1995; Nussbaum, 2007). 
Frente a este planteamiento, otros autores y autoras como Fraser (2008) o Young (2011b) creen que no es suficiente quedarse en la mera redistribución de bienes y destacan el planteamiento de Justicia como Reconocimiento y Participación.

La Justicia Ambiental es definida por Teresa Vicente Giménez (2002, p. 59) como la necesidad de "elaboración de un modelo de Justicia más adecuado a los planteamientos y dinámicas de la ordenación justa del cosmos ecológico". Bryant (1995), por su parte, señala que la Justicia Ambiental "se refiere a las normas y los valores culturales, reglas, reglamentos, conductas, políticas y decisiones de apoyo a comunidades sostenibles, donde la gente puede interactuar con la confianza de que su entorno es seguro, cuidado y protegido" (p. 6).

En este trabajo se da especial importancia a la sostenibilidad, a partir de su interrelación entre los tres pilares que lo componen: el económico, el ambiental y el social (Brundtland, 1992; Middleton y O’Keefe, 2001). La sostenibilidad es definida por la World Commission on Environment and Development (1987) como el "desarrollo que satisface las necesidades del presente, sin comprometer la capacidad de las generaciones futuras para satisfacer sus propias necesidades" (p. 8). A su vez, "no debe poner en peligro los sistemas naturales que sustentan la vida en la Tierra: la atmósfera, las aguas, los suelos, y los seres vivos" (World Commission on Environment and Development, 1987, p. 45).

Aunque algunos autores y autoras, como Dobson (1998), señalan que el movimiento de Justicia no es sobre sostenibilidad, porque busca compartir el riesgo por igual, no deshacerse del riesgo por completo. Sin embargo, nuestro concepto de Justicia Social y Ambiental recoge el enfoque de sostenibilidad, buscando deshacerse del riesgo y llegar a conceptos como el de "crecimiento cero" o el "decrecimiento" (Lawn, 2010; Schneider, Kallis y Martínez-Alier, 2010).

Se trata de reconocer la interdependencia, alrededor de la sostenibilidad, de la Justicia Social, del bienestar económico y de la ordenación ambiental (Agyeman, 2005, 2007; Agyeman, Bullard y Evans, 2003; Haughton, 1999). Un ejemplo de esta interconexión lo refleja Haughton (1999) cuando señala que la dimensión social es crucial, ya que una sociedad injusta socialmente es poco probable que sea una sociedad ambientalmente o económicamente sostenible a largo plazo (y viceversa). Sachs (1996) utiliza el término "EcoJusticia" como las reivindicaciones de un desarrollo respetuoso con las personas y con el ambiente.

En la actualidad también existen otros criterios que han relacionado cuestiones sociales y ambientales, como son los conceptos de impacto ambiental, deuda ecológica y huella ecológica (Altvater, 2011; Martínez-Alier y Oliveras, 2003; Naredo y Valero, 1999; Wackernagel y Rees, 2001) y que son herramientas de acción y medición muy útiles para entender y promover el concepto de Justicia Social y Ambiental (Agyeman, 2005).

Por su parte, el concepto de Justicia Social está integrado en discursos históricamente construidos con un carácter ideológico no exento de conflicto (Rizvi, 1998). Griffiths (2003, p. 55) invita a pensar la Justicia como verbo, es decir, un proyecto dinámico, nunca completo, acabado o alcanzado, siempre sujeto a reflexión y mejora (Murillo y HernándezCastilla, 2011).

A partir de Murillo y Hernández-Castilla (2014) definiremos Justicia Social distanciándola de otros términos con los que frecuentemente se ha asociado y que conllevan a la confusión: 
1. No son solo Derechos Humanos. La dignidad de las sociedades implica el estricto cumplimiento de todos y cada uno de los derechos humanos, y una de las primeras obligaciones de los poderes públicos es garantizarlo. Pero es un punto de partida necesario, no un fin. Una sociedad justa es mucho más.

2. No es Igualdad de Oportunidades. Difícilmente podemos quedarnos satisfechos con una sociedad en la que cualquier persona tenga las mismas oportunidades para ser pobre o rica (Dubet, 2011).

3. No es solo distribución equitativa de bienes. Sin desvalorizar este elemento, en la actualidad existen muchas discriminaciones por razón de género, capacidad, cultura, origen étnico y orientación sexual, insostenibles en una sociedad justa. Conceptos como el reconocimiento o la participación son igual de importantes.

4. No existe solo dentro de un Estado-Nación. No solo se debe globalizar el dinero, también la Justicia (Fraser, 2008).

Murillo y Hernández-Castilla (2011), siguiendo a Fraser (2008), señalan que la Justicia Social se puede recoger en un concepto multidimensional basado en la Redistribución, el Reconocimiento y la Participación. De esta manera se recogerían prácticamente todos los aspectos abordados en el concepto de Justicia Social y Ambiental.

A su vez, coincidimos con Young (2011) en su interpretación de que la dificultad para abordar las injusticias reside en que tiene un fuerte componente estructural.

La injusticia estructural existe cuando los procesos sociales sitúan a grandes grupos
de personas bajo la amenaza sistemática del abuso o de la privación de los medios
necesarios para desarrollar o ejercitar sus capacidades, al mismo tiempo que estos
procesos capacitan a otros para abusar o tener un amplio espectro de oportunidades
para desarrollar y ejercitar capacidades a su alcance (...). La injusticia estructural se
da como consecuencia de muchos individuos e instituciones que actúan para perseguir
sus metas e intereses particulares, casi siempre dentro de los limites de normas y leyes
aceptadas. (Young, 2011, p. 69)

Desde nuestra definición, coincidiendo con Bosselmann (2006), tendremos en cuenta la Justicia intergeneracional (entre generaciones), intrageneracional (dentro de cada generación), e interespecies (entre las especies). La Justicia entre naciones (Nussbaum, 2007), la incluimos dentro de todas ellas, porque entendemos que en material social y ambiental las fronteras no deben existir.

Además, damos importancia al concepto de dignidad de Platón (380 a.C./2005) y de Kant (1781/2005) como valor inherente al ser humano en cuanto ser racional, dotado de libertad y autonomía para cambiar, mejorar y desarrollar su vida plenamente (Sanz y Serrano, 2016).

Como todo ello, se puede afirmar que,

La Justicia Social y Ambiental consiste en conseguir integrar el reconocimiento, la participación y la distribución de recursos materiales, culturales y ambientales (tanto perjudiciales como beneficiosos) de forma plena y equitativa, todo ello bajo un criterio intrageneracional, intergeneracional, interespecies e internacional que permita el desarrollo de una vida digna. (Carneros, 2018) 


\section{Educación para la justicia social y ambiental}

Desde esta perspectiva compleja y multidimensional, la conceptualización de la Educación para la Justicia Social y Ambiental no puede tener otras características. Así, de entrada, se puede afirmar que una educación comprometida con la justicia ambiental es inseparable de la lucha política por la justicia social; y viceversa, una autentica educación para la justicia social no puede dejar de lado la lucha por una justicia ambiental. Enfoques aislados se nos antojan insuficientes y, con ello, limitadores de una educación que busque una transformación real (Carneros y Murillo, 2017).

En segundo lugar, es preciso insistir que una Educación PARA la Justicia Social y Ambiental es, de forma necesaria, una educación EN Justicia Social y Ambiental y DESDE la Justicia Social y Ambiental (Murillo y Hernández-Castilla, 2014). Efectivamente, una educación transformadora debe incorporar en sus procesos de enseñanza y aprendizaje competencias, contenidos y valores relacionados con la Justicia Social y Ambiental. Así, es necesario que la educación aborde aspectos tales como desde comprender la historia de los grupos minoritarios que han sido tradicionalmente excluidos, conocer cauces de participación institucional o desarrollar la competencia de participar en debates constructivos, hasta conocer los procesos de degradación medioambiental y de destrucción de recursos naturales o saber reciclar materiales (Boyle-Baise, 2003). Pero también es necesario que la educación se desarrolle en entornos justos y medioambientalmente sostenibles. Una educación democrática, que distribuya apoyos en función de necesidades y una educación en escuelas sostenibles, que gestionen bien su energía y reciclen, son aspectos esenciales. Es la coherencia entre el decir y el hacer.

Como hemos señalado, la perspectiva multidimensional de la Justicia Social y Ambiental nos llevan a pensar en una educación de carácter igualmente multidimensional, en este caso con cuatro dimensiones interdependientes e interrelacionadas:

1. Educación equitativa (inclusiva)

2. Educación crítica (e innovadora)

3. Educación democrática

4. Educación para el desarrollo sostenible (o ambiental)

Efectivamente, una Educación para (en y desde) la Justicas Social y Ambiental ha de ser en primer lugar una Educación Equitativa, de todos y todas, para todos y todas y con todos y todas. Una educación, por donde todos estén, se desarrollen y participen, es decir, una Educaciónj Inclusiva. Una educación donde haya políticas y hechos de redistribución de apoyos y recursos que logren compensar las diferencias de partida y logren que todos se desarrollen al máximo de su potencialidad.

En palabras de Antonio Bolívar (2005), una educación equitativa:

Sería aquella que trata a todos los alumnos como iguales y que intenta favorecer una sociedad equitativa, en la que los bienes esenciales están distribuidos conforme a las reglas de la justicia y que favorece la cooperación en un plano de igualdad" (Crahay y otros, 2003:12). Esto supone, en primer lugar, que determinados bienes educativos sean distribuidos de forma equitativa, por ejemplo, los recursos, la calidad del profesorado, la oferta educativa, cuidando que las desigualdades no condicionen el aprendizaje y el rendimiento escolar, y poniendo los medios y recursos compensatorios en los alumnos desfavorecidos natural o socialmente. (pp. 43-44) 
Una educación que fomenta el reconocimiento y valoración explícita de las diferencias y características de cada uno de los y las estudiantes a través de una atención diferencial de sus necesidades.

En segundo lugar, una Educación Crítica. Una educación para la Justicia Social y Ambiental requiere hacer de los y las estudiantes agentes da cambio social y a que los y las docentes sean intelectuales críticos (Smyth, 2011), dos de los elementos de la educación crítica.

Siguiendo a Henry A. Giroux (2003), la Educación Crítica es:

Un intento deliberado de influir en cómo y qué conocimiento e identidades se producen en cada relación de poder y contexto particular. [...] relacionando las personas con la vida pública, la responsabilidad social o las exigencias de una ciudadanía crítica" [...], siendo central ofrecer guía sobre asuntos de igualdad, libertad y justicia para que los estudiantes puedan identificar problemas sociales, raciales y las inequidades de clase que las animan. (p. 153)

La finalidad de esta educación, por tanto, es la transformación y emancipación social a través la generación de una conciencia crítica en los estudiantes (Giroux y Shannon, 1997, 2003; Kincheloe, 2008).

Continuando con las ideas de Giroux (1990), el docente es un intelectual que puede convertir la enseñanza es un elemento crítico. El autor propone unos determinados pasos para lograr una pedagogía crítica: buscar el problema, contextualizarlo, darle un tratamiento cultural (analizarlo de forma interdisciplinar y teniendo en cuenta la cultura dominante en el que se enmarca), buscar las estrategias de aprendizaje más adecuadas, proponer un texto alternativo e incluir las experiencias vitales de los estudiantes en este texto. Es decir, una educación innovadora.

El tercer elemento es que sea una Educación Democrática (Belavi y Murillo, 2016; Biesta, 2015; Dewey, 2016/1995; Gutmann, 1987). En palabras de Mursell (1955, p. 3) formuladas hace más de 60 años: "si la educación no es democrática es peligrosa y por lo tanto no tiene razón de ser".

Así, una escuela verdaderamente democrática debe caracterizarse por:

- Favorece que todos y cada uno participe en condiciones de igualdad en la toma de decisiones en los asuntos que le afecten, bien sea de carácter curricular, organizativo, institucional o de cualquier índole.

- Promueve la igualdad estructural, entendida como la no existencia de relaciones de poder ni de desigualdad.

- Favorece fuertes relaciones entre la escuela y la comunidad.

- Crear comunidades profesionales de aprendizaje teniendo en cuenta la diversidad de los estudiantes, así como un proyecto compartido de cooperación y colaboración.

- Hay un planteamiento explícito de impactar en la sociedad, no sólo denunciar las situaciones de injusticia sino cambiarlas.

- El currículum debe generar conocimiento, significado, por lo que ningún grupo puede reclamar la propiedad exclusiva del conocimiento. 
Por último, es necesario hacer explícito la implicación de la Educación en la Justicia Ambiental. Con términos tales como Educación para el Desarrollo Sostenible (Macedo y Salgado, 2007), Educación para la conciencia planetaria de la ciudadanía (Novo y Murga, 2010), o Educación Ambiental, pero no entendido como una materia, sino como un enfoque de la educación. Sería, como señala Gadotti (2005), una educación para una ciudadanía planetaria que conlleve una filosofía educativa enfocada tanto en lo local como lo global, respetando ambos contextos a través de la cooperación la solidaridad, la economía solidaria y una vida sostenible con el mundo.

\section{Referencias}

Agyeman, J. (2005). Sustainable communities and the challenge of environmental justice. Nueva York, NY: New York University Press.

Agyeman, J. (2007). Environmental justice and sustainability. En M. Atkinson, S. Dietz y E. Neumayer (Eds.), Handbook of sustainable development (pp. 171-188). Cheltenham: Edward Elgar. https://doi.org/10.4337/9781847205223.00020

Agyeman, J. y Evans, B. (2004). Just sustainability: The emerging discourse of environmental justice in Britain? The Geographical Journal, 170(2), 155-164. https://doi.org/10.1111/j.0016-7398.2004.00117.x

Agyeman, J., Bullard, R. D. y Evans, B. (2003). Just sustainabilities: Development in an unequal world. Boston, MA: MIT press.

Altvater, E. (2011). Los límites del capitalismo. Acumulación, crecimiento y huella ecológica. Buenos Aires: Mar Dulce.

Avedaño, M. (2010). La paridad participativa en la obra de Nancy Fraser. Aequalitas: Revista Jurídica de Igualdad de Oportunidades entre Mujeres y Hombres, 26, 58-70.

Beauchamp, T. L. (2001). Philosophical ethics: An introduction to moral philosophy. Londres: McGrawHill Book Company.

Belavi, G. y Murillo, F.J. (2016). Educación, democracia y justicia social. Revista Internacional de Educación para la Justicia Social (RIEJS), 5(1), 13-34.

Bell, L. A. (2007). Theoretical foundations for social justice education. En L. A. Adams, L. A. Bell y P. Griffin (Eds.), Teaching for diversity and social justice: A sourcebook (pp. 3-15). Nueva York, NY: Routledge.

Benedicto, R. (2010). Liberalismo y comunitarismo. Un debate inacabado. Studium: Revista de Humanidades, 16, 201-229.

Biesta, G. J. J. (2015). Beyond learning. Democratic education for a human future. Boulder, CO: Paradigm Publishers.

Bolívar, A. (2005). Equidad educativa y teorías de la justicia. REICE. Revista Iberoamericana sobre Calidad, Eficacia y Cambio en Educación, 3(2), 42-69.

Bosselmann, K. (2006). Ecological justice and law. En B. J. Richardson y S. Wood (Eds.), Environmental law for sustainability (pp. 129-163). Oxford: Hart Publishing.

Boyle-Baise, M. (2003). Doing democracy in social studies methods. Theory and Research in Social Education, 31(1), 50-70. https://doi.org/10.1080/00933104.2003.10473215

Brundtland, G. H. (1992). Nuestro futuro común. Madrid: Alianza.

Bryant, B. (1995). Environmental justice. Issues, policies, and solutions. Washington, DC: Island Press. 
Bullard, R. D. (1993a). The threat of environmental racism. Natural Resources and Environment, 7(3), 23-26.

Bullard, R. D. (1993b). Confronting environmental racism: Voices from the grassroots. Boston, MA: South End Press.

Carneros, S. (2018). La escuela alternativa: Un modelo en búsqueda de la justicia social y ambiental. Tesis doctoral. Universidad Autónoma de Madrid.

Carneros, S. y Murillo, F. J. (2017). Aportaciones de las escuelas alternativas a la justicia social y ambiental: Autoconcepto, autoestima y respeto. REICE. Revista Iberoamericana sobre Calidad, Eficacia y Cambio en Educación, 15(3), 129-156.

Chavis, B. F. (1993). Forward. En R. D. Bullard (Ed.), Confronting environmental racism: Voices from the grassroots (pp. 3-5). Boston, MA: South End Press.

Collins, P. H. (1991). Fighting words: Black women and the search for justice. Nueva York, NY: Routledge.

Cortina, A. (1993). Ética aplicada y democracia radical. Madrid: Tecnos.

Dewey, J. (1916/1995). Democracia y educación. Madrid: Morata

Dobson, A. (1998). Justice and the environment conceptions of environmental sustainability and theories of distributive justice. Oxford: Oxford University Press. https://doi.org/10.1093/0198294956.001.0001

Dubet, F. (2011). Repensar la justicia social. Contra el mito de la igualdad de oportunidades. Buenos Aires: Siglo XXI Editores.

Dworkin, R. (1981). What is equality? Part 2: Equality of resources. Philosophy and Public Affairs, $10(4), 283-345$.

Dworkin, R. (1985). A matter of principle. Oxford: Clarendon Press.

Dworkin, R. (1993). Ética privada e igualitarismo político. Barcelona: Paidós.

Fraser, N. (1997). Iustitia Interrupta. Reflexiones críticas sobre la posición "postsocialista". Bogotá: Siglo del hombre editores.

Fraser, N. (2008). Escalas de justicia. Barcelona: Herder.

Fraser, N. y Honneth, A. (2006). ¿Redistribución o reconocimiento? Madrid: Morata.

Gadotti, M. (2005). Pedagogia da terra e cultura de sustentabilidade. Revista Lusófona de Educação, $6,15-29$

Giroux, H.A. (1990). Los profesores como intelectuales. Barcelona: Paidós.

Giroux, H.A. (2003). Pedagogía y política de la esperanza. Buenos Aires: Amorrortu.

Giroux, H.A. y Shannon, P. (1997). Education and cultural studies: Toward a performative practice. Nueva York, NY: Routledge.

Gosseries, A. (2012). La cuestión generacional y la herencia Rawlsiana. Revista del Instituto de Investigaciones Jurídicas y Sociales Ambrosio Lucas Gioja, 6(8), 71-90.

Grass, R. (1995). Environmental education and environmental justice: A three circles perspective. Pathways to Outdoor Communication, 5, 9-13.

Griffiths, M. (2003). Action for social justice in education fairly different. Maidenhead: Open University Press.

Gutmann, A. (1987). Democratic education. Princeton, NJ: Princeton University Press. 
Harnett, D. (2001, enero). The history of justice. Conferencia presentada en el Social justice forum. Chicago, IL: Loyola University.

Haughton, G. (1999). Environmental justice and the sustainable city. JPER: Journal of Planning Education and Research, 18(3), 233-243. https://doi.org/10.1177/0739456X9901800305

Hayek, F. (1948). Individualism and Economics Order. Chicago, IL: University of Chicago Press.

Heiman, M. K. (1996). Race, waste, and class: New perspectives on environmental justice. Antipode, 28(2), 111-121. https://doi.org/10.1111/j.1467-8330.1996.tbo0517.x

Hervé, D. (2010). Noción y elementos de la justicia ambiental: Directrices para su aplicación en la planificación ambiental y en la evaluación ambiental estratégica. Revista de Derecho, 23(1), 936.

Hoerner, J. A. y Robinson, N. (2008). A climate of change. Oakland, CA: Environmental Justice and Climate Change Initiative (EJCC).

Hofrichter, R. (1994). Toxic struggles: The theory and practice of environmental justice. Philadelphia, PA: New Society Publishers.

Honneth, A. (2007). Reificación: Un estudio en la teoría del reconocimiento. Buenos Aires: Katz.

Iglesias, C. (2012). Justicia como redistribución, reconocimiento y representación: Las reconciliaciones de Nancy Fraser. Investigaciones Feministas, 3, 251-269.

Jost, J. T. y Kay, A. C. (2010). Social justice: History, theory, and research. En S. T. Fiske, D. T. Gilbert y L. Gardner (Eds.), Handbook of social psychology (pp. 1122-1165). Hoboken, NJ: Wiley. https://doi.org/10.1002/9780470561119.socpsy002030

Kant, I. (1781/2005). Crítica de la razón pura. Madrid: Taurus.

Kincheloe, J.L. (2008). Critical pedagogy primer. Nueva York, NY: Peter Lang.

Lawn, P. (2010). Facilitating the transition to a steady-state economy: Some macroeconomic fundamentals. Ecological Economics, $\quad 69(5), \quad$ 931-936. https://doi.org/10.1016/j.ecolecon.2009.12.013

Macedo, B. y Salgado, C. (2007). Educación ambiental y educación para el desarrollo sostenible en América Latina. Revista de la Cátedra Unesco sobre Desarrollo Sostenible, 29, 29-37.

Macintyre, A. C. (1984). Tras la virtud. Barcelona: Crítica.

Martínez-Alier, J. y Oliveras, A. (2003). ¿Quién debe a quién? Deuda ecológica y deuda externa. Barcelona: Icaria.

Middleton, N. y O’Keefe, P. (2001). Redefining sustainable development. Londres: Pluto Press.

Mosterín, J. y Riechmann, J. (1995). Animales y ciudadanos. Indagación sobre el lugar de los animales en la moral y en el derecho de las sociedades industrializadas. Madrid: Talasa.

Murillo, F. J. y Hernández-Castilla, R. (2011). Trabajar por la justicia social desde la educación. REICE. Revista Electrónica Iberoamericana sobre Calidad, Eficacia y Cambio en Educación, 9(4), 3-6.

Murillo, F. J. y Hernández-Castilla, R. (2014). Liderando escuelas justas para la justicia social. Revista Internacional de Educación para la Justicia Social, 3(2), 13-32.

Mursell, J. L. (1955). Principles of democratic education. Nueva York, NY: Norton.

Nagel, T. (1991). Equality and partiality. Oxford: Oxford University Press.

Naredo, J. M. y Valero, A. (1999). Desarrollo económico y deterioro ecológico. Madrid: Fundación Argentaria-Visor. 
North, C. E. (2006). More than words? Delving into the substantive meaning (s) of "social justice" in education. Review of Educational Research, 76(4), 507-535. https://doi.org/10.3102/00346543076004507

Novo, M. y Murga, M. A. (2010). Educación ambiental y ciudadanía planetaria. Revista Eureka sobre $\begin{array}{llllll}\text { Enseñanza Divulgación } \quad y \quad \text { de las } & \text { Ciencias, } & 7, & 179-186 .\end{array}$ https://doi.org/10.25267/Rev_Eureka_ensen_divulg_cienc.2010.v7.iextra.03

Nozick, R. (1988). Anarquía, estado y utopía. Ciudad de México: Fondo de Cultura Económica.

Nussbaum, M. C. (2002). Las mujeres y el desarrollo humano: El enfoque de las capacidades. Madrid: Herder.

Nussbaum, M. C. (2004). Beyond the social contract: Capabilities and global justice. Oxford Development Studies, 32(1), 3-18.

Nussbaum, M. C. (2007). Las fronteras de la Justicia: Consideraciones sobre la exclusión. Madrid: Paidós.

Platón. (380 a.C./2005). La república. Madrid: Alianza Editorial.

Rawls, J. A. (1979). Teoría de la justicia. Madrid: Fondo de Cultura Económica.

Rawls, J. A. (1993). Political liberalism. Nueva York, NY: Columbia University Press.

Rawls, J. A. (2002). La justicia como equidad. Una reformulación. Barcelona: Paidós.

Raz, J. (1986). The morality offreedom. Oxford: Clarendon Press.

Riechmann, J. (2000). Un mundo vulnerable. Ensayos sobre ecología, ética y tecnociencia. Madrid: Los Libros de la Catarata.

Riechmann, J. (2003). Tres principios básicos de justicia ambiental. Revista Internacional de Filosofía Política, 21, 103-120.

Riechmann, J., Naredo, J. M., Bermejo, R., Estevan, A., Taibo, C., Rodriguez, J. C., . . Nieto, J. (1995). De la economía a la ecología. Madrid: Trotta.

Rizvi, F. (1998). Some thoughts on contemporary theories of social justice. En B. Atweh, S. Kemmis y P. Weeks (Eds.), Action research in practice: Partnerships for social justice in education (pp. 4756). Londres: Routledge.

Rodríguez Zepeda, J. (2009). El principio rawlsiano de diferencia: Dilemas de interpretación. Enrahonar, 43, 31-59. https://doi.org/10.5565/rev/enrahonar.276

Sachs, A. (1996). Ecojusticia. La unión de los derechos humanos y el medio ambiente. Bilbao: Cuadernos World Wacht.

Sandel, M. J. (1982). El liberalismo y los límites de la justicia. Barcelona: Gedisa.

Sanz, J. y Serrano, A. (2016). El desarrollo de capacidades en la educación. Una cuestión de justicia social. Sinéctica, 8(46), 1-16.

Schlosberg, D. (2007). Defining environmental justice. Theories, movements, and nature. Oxford: Oxford University Press. https://doi.org/10.1093/acprof:oso/9780199286294.001.0001

Schneider, F., Kallis, G. y Martínez-Alier, J. (2010). Crisis or opportunity? Economic degrowth for social equity and ecological sustainability. Introduction to this special issue. Journal of Cleaner Production, 18(6), 511-518. https://doi.org/10.1016/j.jclepro.2010.01.014

Sen, A. (1992). Inequality reexamined. Cambridge, MA: Harvard University Press.

Sen, A. (1995). Nuevo examen a la desigualdad. Madrid: Alianza.

Sen, A. (2010). La idea de justicia. Madrid: Taurus. 
Smyth, J. (2011). Critical pedagogy for social justice. Londres: Continuum.

Snyder, G. (1995). A place in space. Washington, DC: Counterpoint.

Taylor, C. (2003). Cross-purposes: The liberal-communitarian debate. En D. Matravers y J. E. Pike (Eds.), Debates in contemporary political philosophy: An anthology (pp. 195-212). Nueva York, NY: Routledge.

Trejo Pérez, P. (2016). Reflexiones desde la banca. Naucalpan de Juárez: Innovaciones Editorial Lagares.

Valcárcel, A. (2002). Ética para un mundo global: una apuesta por el humanismo frente al fanatismo. Madrid: Temas de Hoy.

Vicente Giménez, T. (2002). Justicia ecológica y protección del medio ambiente. Barcelona: Trotta.

Wackernagel, M. y Rees, W. E. (2001). Nuestra huella ecológica: Reduciendo el impacto humano sobre la tierra. Santiago de Chile: LOM Ediciones.

Walzer, M. (2001). Las esferas de la justicia: Una defensa del pluralismo y la igualdad. Ciudad de México: Fondo de Cultura Económica.

Weiss, E. B. (1999). Un mundo justo para las futuras generaciones: Derecho internacional, patrimonio común y equidad intergeneracional. Madrid: Ediciones Mundi-Prensa.

Wissenburg, M. (1999). An Extension of the Rawlsian Savings Principle to Liberal Theories of Justice in General. En A. Dobson (Ed.), Fairness and futurity: Essays on environmental sustainability and environmental (pp. 173-198). Oxford: Oxford University Press. https://doi.org/10.1093/0198294891.003.0008

World Commission on Environment and Development. (1987). Our common future. Oxford: Oxford University Press.

Young, I. M. (2000a). Inclusion and democracy. Oxford: Oxford University Press.

Young, I. M. (2000b). La justicia y la política de la diferencia. Madrid: Cátedra.

Young, I. M. (2011). Responsabilidad por la justicia. Madrid: Morata. https://doi.org/10.1093/acprof:oso/9780195392388.001.0001

\section{Breve CV de los autores}

\section{Sergio Carneros}

Doctor en Educación por la Universidad Autónoma de Madrid. Diplomado en Magisterio de Educación Física (obteniendo el Premio Extraordinario) y Licenciado en Psicopedagogía por la Universidad de Alcalá de Henares (UAH). Máster Oficial en Neurocontrol Motor por la Universidad Rey Juan Carlos (URJC) y Postgrado de Experto en Atención Temprana por la Universidad Autónoma de Madrid (UAM). Es funcionario de carrera del Cuerpo de Maestros y miembro del grupo de investigación Cambio Educativo para la Justicia Social (GICE). Funda y preside la Red Internacional de Educación (RIE). Ha trabajado en centros públicos de Educación Infantil y Primaria y de Educación Especial en España y coordina proyectos educativos con gobiernos, escuelas y universidades en países como El Salvador, Panamá, Filipinas, Bolivia o Ecuador. Realiza decenas de formaciones y ponencias anuales y cuenta con diversas publicaciones de libros. ORCID ID: 0000-0003-0275-735X. Email: sergio.carneros@hotmail.com 


\section{F. Javier Murillo}

Profesor titular del Área de Métodos de Investigación y Diagnóstico en Educación de la Universidad Autónoma de Madrid. Coordinador del Doctorado en Educación de la UAM. Coordinador del grupo de Investigación "Cambio Educativo para la Justicia Social" (GICE) y Secretario Académico del Instituto de Derechos Humanos, Democracia y Cultura de Paz y No Violencia (DEMOSPAZ) de la UAM. Fue Coordinador General del Laboratorio Latinoamericano de Evaluación de la Calidad de la Educación (LLECE), de la UNESCO, y Director de Estudios del Centro de Investigación y Documentación Educativa (CIDE) del Ministerio de Educación de España. Es Coordinador de la Red Iberoamericana de Investigación sobre Cambio y Eficacia Escolar (RINACE), Director de REICE. Revista Iberoamericana sobre Calidad, Eficacia y Cambio en Educación, de la Revista Iberoamericana de Evaluación Educativa y de la Revista Internacional de Educación para la Justicia Social. Página de docencia: www.fjaviermurillo.es. ORCID ID: 0000-0002-8003-4133. Email: javier.murillo@uam.es

\section{Irene Moreno-Medina}

Doctoranda en Educación en la Universidad Autónoma de Madrid. Licenciada en Psicopedagogía y Diplomada en Educación Social (ambas por la Universidad de Málaga). Miembro de Grupo de Investigación "Cambio Educativo para la Justicia Social" (GICE), de la Universidad Autónoma de Madrid. Investigando sobre la cultura de los centros en contextos desafiantes. ORCID ID: 0000-0002-3745-7253. Email: irene.morenomedina@estudiante.uam.es 